\title{
Left Atrial Thrombus Present: Is That about It?
}

\author{
Jitin Narula ${ }^{1}$ Neeraj Kumar ${ }^{1}$ Sanjay Kumar ${ }^{1}$ Saurabh Jaiswal ${ }^{2}$ Ritwick Raj Bhuyan ${ }^{2}$ \\ ${ }^{1}$ Department of Cardiac Anaesthesiology, Max Heart and Vascular \\ Institute, Max Super Specialty Hospital, Patparganj, New Delhi, India \\ ${ }^{2}$ Department of Cardiovascular Surgery, Max Heart and Vascular \\ Institute, Max Super Specialty Hospital, Patparganj, New Delhi, India

\begin{abstract}
Address for correspondence Jitin Narula, DM, Department of Cardiac Anaesthesiology, Max Heart and Vascular Institute, Max Super Specialty Hospital, Plot No. 108A, Indraprastha Extension, Patparganj, New Delhi 110092, India (e-mail: jatin.narula.13@gmail.com).
\end{abstract}

\begin{abstract}
Keywords

- left atrial thrombus

- embolization

- thrombus dislodgement

- transesophageal echocardiography

Two-dimensional echocardiography is extremely useful in detecting localized as well as floating intracardiac bodies. Transesophageal echocardiography (TEE) is highly sensitive in localizing intracardiac masses especially those present in the posterior structures of the heart, such as the left atrium (LA) and the left atrial appendage (LAA), which are extremely difficult to image using the transthoracic echocardiography. Dislodgement of intracardiac masses (thrombus, tumor) can be associated with high risk of life-threatening hemodynamic perturbations or embolic phenomenon. Cardiac manipulations should thus be minimized in patients with intracardiac masses. We discuss the case of LAA thrombus dislodgement during inferior vena cava cannulation. Intraoperative TEE demonstrated a fixed thrombus in the LAA, and despite careful attention by the surgeon, trivial cardiac manipulation during inferior vena cava cannulation leads to the dislodgment of the LAA thrombus and its free floatation in the LA. This case report highlights the crucial role of a vigilant TEE examination in patients with intracardiac thrombi. Identification of echocardiographic risk factors for prediction of dislodgement of LAA is of utmost importance to avoid inadvertent complications.
\end{abstract}

\section{Introduction}

The left atrium (LA) and its appendage are common sites for the formation of thrombi in patients with mitral stenosis and atrial fibrillation due to the stasis of blood or a poorly contracting LA. The left atrial thrombus may break into several smaller pieces forming free-floating masses, leading to catastrophic embolic events such as a stroke, systemic embolization, and total or partial obstruction of the left ventricular inflow. ${ }^{1-4}$ Two-dimensional (2D) echocardiography is not just extremely sensitive in detecting thrombi of the LA, but it is also predictive of a possibility of a thrombus formation by the detection of a spontaneous echo contrast. $^{5-7}$ Difficulties in the visualization of the left atrial appendage (LAA) using transthoracic echocardiography (TTE) have been easily overcome with the advent of transesophageal echocardiography (TEE), which has near $100 \%$ sensitivity in detection of left atrium and left atrial appendage thrombi. Because of their fragility, intracardiac thrombi are at extremely high risk of dislodgement and embolization, leading to adverse sequelae. This can easily happen with any cardiac manipulation that may occur during caval cannulation, insertion of cardioplegia catheter, or application of aortic cross-clamp. In this case, TEE instantaneously detected the dislodged thrombus following which early bypass was initiated and adverse sequelae averted.

\section{Case Report}

A 36-year-old woman who presented with the New York Heart Association (NYHA) III dyspnea was found to have severe rheumatic mitral stenosis on TTE and was under evaluation for possible need of mitral valve replacement. The patient had chronic atrial fibrillation and was receiving oral anticoagulation. She did not have a history of transient ischemic attack or stroke. Preoperative TTE revealed severe calcific mitral stenosis, a left ventricular ejection fraction of $55 \%$, moderate pulmonary artery hypertension, mild
DOI https://doi.org/ $10.1055 / \mathrm{s}-0037-1604172$.
Copyright ( 2017 Official Publication of License terms The Simulation Society (TSS), accredited by International Society of Cardiovascular Ultrasound (ISCU) 


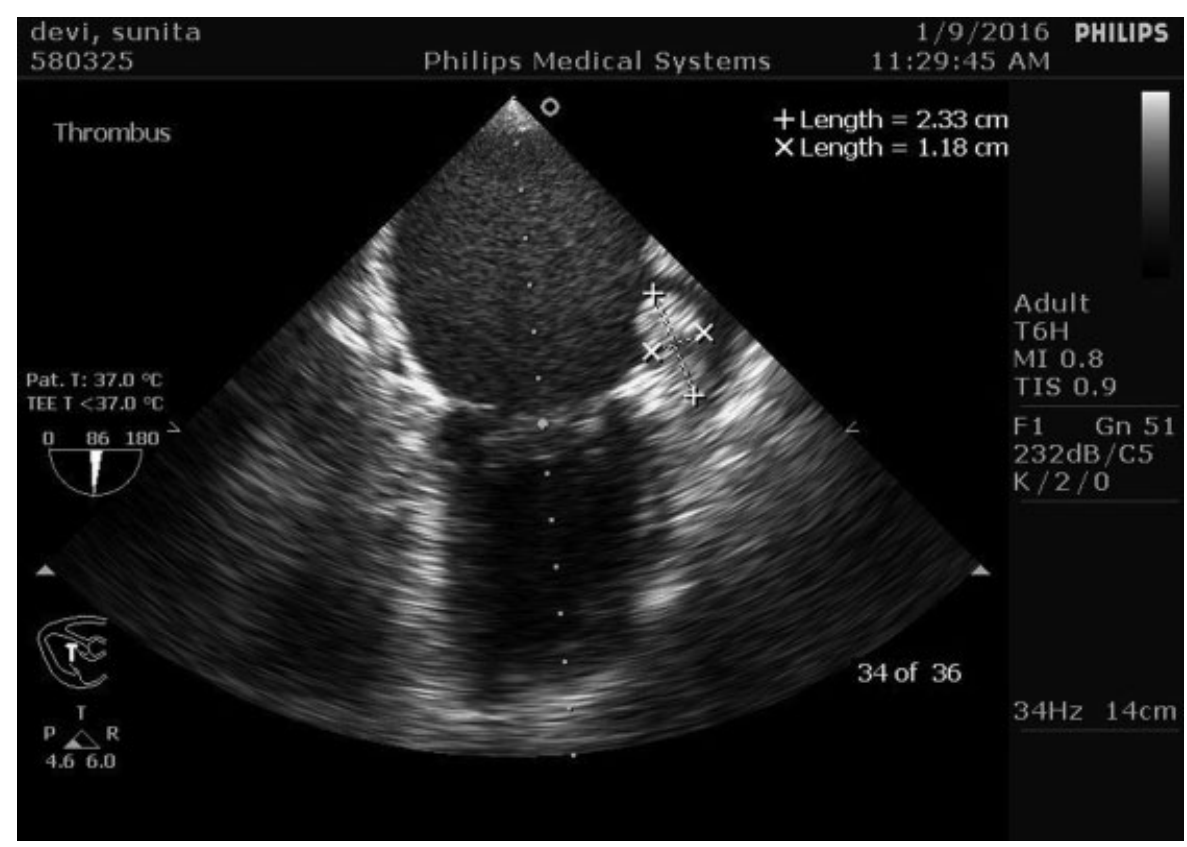

Fig. 1 Midesophageal two-chamber view showing thrombus in the left atrial appendage measuring $2.33 \times 1.18 \mathrm{~cm}$.

tricuspid regurgitation, mildly hypokinetic right ventricle, and left atrial (LA) spontaneous echo contrast. The LAA could not be visualized on TTE.

TEE confirmed findings of TTE and showed an LAA thrombus $(2.33 \times 1.18 \mathrm{~cm})$, well-circumscribed, spherical, mobile echodensity within the ostium of the LAA ( - Fig. 1, - Video 1 ). After systemic heparinization, aortic and superior vena cava cannulations were uneventfully accomplished. However, during an attempt to cannulate the inferior vena cava (IVC), the LA thrombus was seen to pop out of the LAA and freely float in the LA (-Fig. 2, - Video 2). The freely mobile thrombus bounced against the thickened and tightly stenotic mitral valve ( - Fig. 3 ,
- Video 3). The patient was immediately put in a steep Trendelenburg's position, IVC was cannulated, and cardiopulmonary bypass was instituted to drain out the cardiac chambers. As the heart was drained into the reservoir, the dislodged thrombus was visualized getting cornered in the LA near the left upper pulmonary vein ostium ( - Video 4 ). The dislodged clot was continuously visualized until the aortic cross-clamp was applied, and even though the stenotic mitral valve was protective in preventing the embolization of the large ballvalve thrombus, any associated small fragment could have made way into the systemic circulation. It was subsequently retrieved after cardioplegic cardiac arrest (-Fig. 4).

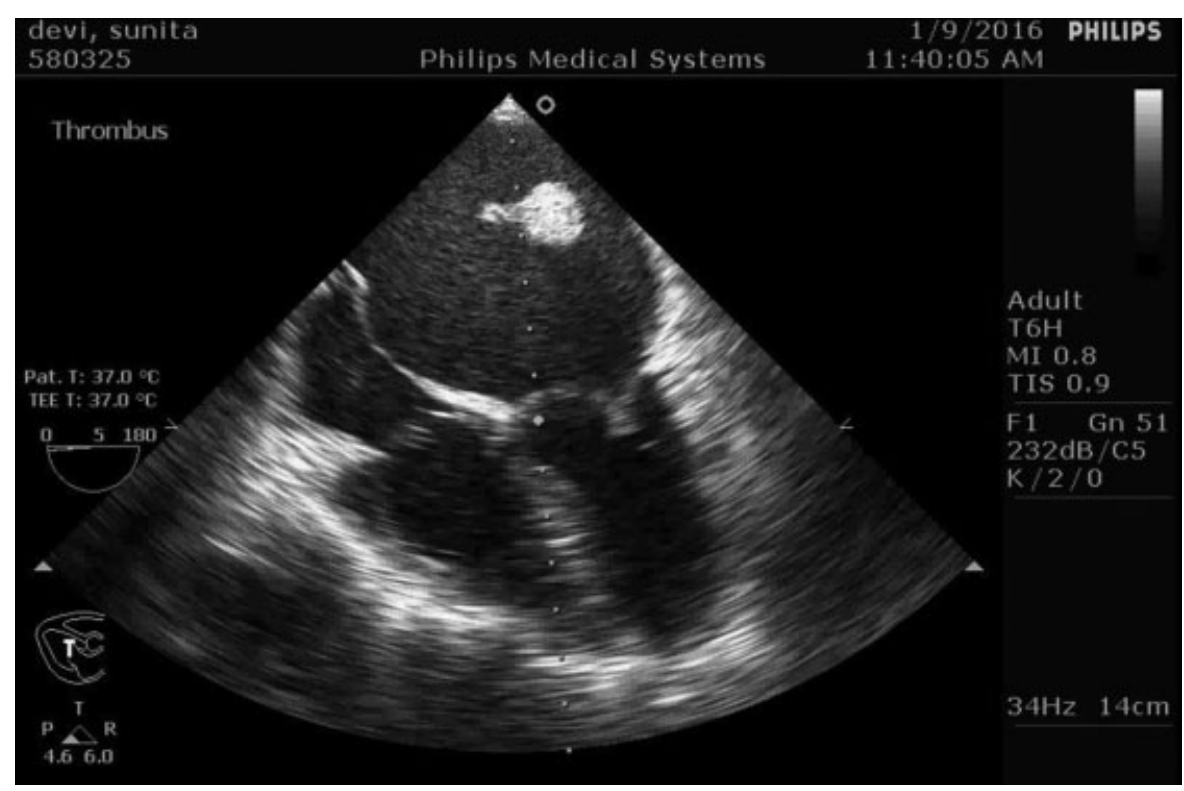

Fig. 2 Midesophageal four-chamber view showing the dislodged thrombus freely floating in the left atrium. 


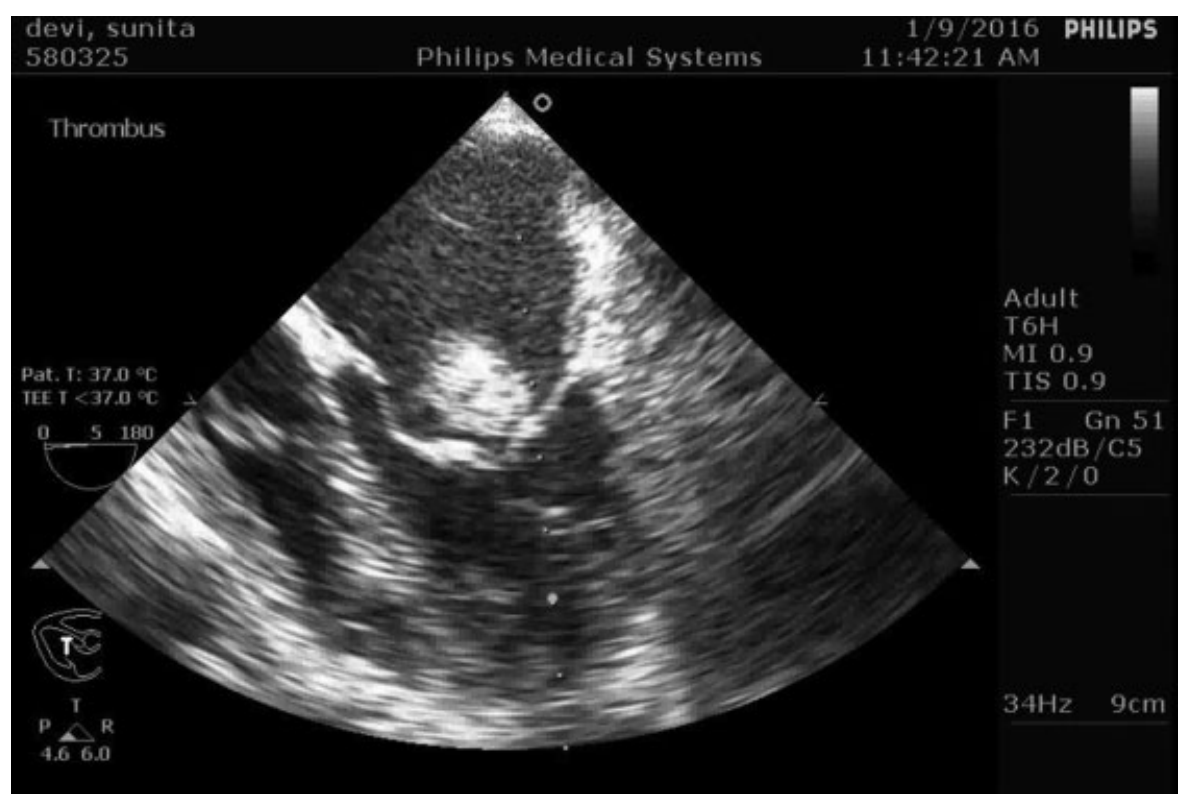

Fig. 3 Midesophageal four-chamber view showing free-floating mobile thrombus bouncing against the thickened and tightly stenotic mitral valve.

\section{Video 1}

Midesophageal two-chamber view showing thickened, calcified, and stenotic mitral valve leaflets along with a thrombus in the left atrial appendage measuring $2.33 \times 1.18 \mathrm{~cm}$. Online content including video sequences viewable at: www.thieme-connect. com/ejournals/html/doi/10.1055/s-0037-1604172.

\section{Video 2}

Midesophageal four-chamber view showing the dislodged thrombus freely floating in the left atrium. Online content including video sequences viewable at: www.thieme-connect.com/ejournals/html/doi/ 10.1055/s-0037-1604172.

\section{Video 3}

Midesophageal four-chamber view showing freefloating mobile thrombus bouncing against the thickened and tightly stenotic mitral valve. Online content including video sequences viewable at: www. thieme-connect.com/ejournals/html/doi/10.1055/ s-0037-1604172.

\section{Video 4}

The dislodged thrombus visualized getting cornered in the left atrium near the left upper pulmonary vein ostium after induction of cardiopulmonary bypass. Online content including video sequences viewable at: www.thieme-connect.com/ejournals/html/doi/ $10.1055 / \mathrm{s}-0037-1604172$.

Gross examination of the specimen revealed a spherical mass of $2.5 \mathrm{~cm}$ in diameter, which was subsequently confirmed to be a thrombus (-Fig. 4). The patient was closely monitored in the postoperative period for development of any

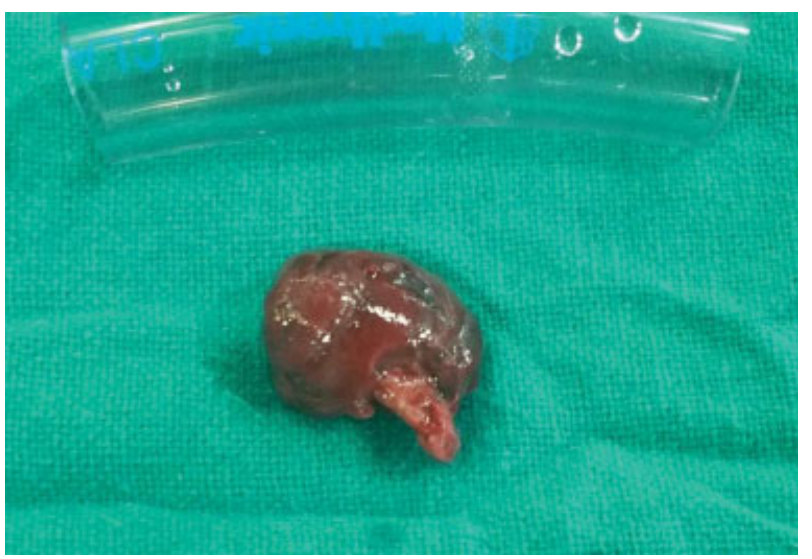

Fig. 4 Organized thrombus as seen upon gross examination. 
Table 1 Stages of thrombus formation

\begin{tabular}{|l|l|}
\hline Stage & Features \\
\hline I Stasis & $\begin{array}{l}\text { Stasis of blood, a predisposing factor to thrombus formation, manifested as intracardiac "smoke" or } \\
\text { spontaneous echo contrast and indicative of erythrocyte rouleaux formation }\end{array}$ \\
\hline II Sludge & $\begin{array}{l}\text { Sludge (very dense smoke) has an echocardiographic appearance that is more viscid than smoke but less } \\
\text { dense than thrombus }\end{array}$ \\
\hline III Thrombus & $\begin{array}{l}\text { Complete thrombus formation; attached by a pedicle to the left atrial appendage or may be in the form of } \\
\text { layered clots that may be early or organized }\end{array}$ \\
\hline
\end{tabular}

adverse embolic outcome and was uneventfully discharged from the intensive care unit (ICU) on postoperative day 3.

\section{Discussion}

This case report highlights the pivotal role TEE plays in patients with diagnosed or are suspected to have intracardiac left atrial masses. Two-dimensional (2D) echocardiography is extremely helpful in determining the nature, size, spatial orientation of masses that most commonly include left atrial thrombus, prolapsing type of left atrial myxoma, papillary fibroelastomas, Lambl's excrescences, and fragments of vegetations. ${ }^{8}$

Stasis of blood as seen in patients with mitral stenosis and the presence of atrial fibrillation or a poorly contracting LA predispose to the formation of thrombi in the LA, more commonly its appendage. Thrombus can be readily identified by TTE with a high specificity. The sensitivity of TTE is, however, inadmissibly low, mostly because atrial thrombi commonly occur in the LAA which is difficult to visualize on TTE. Because of it being located immediately adjacent to the esophagus, the LA is the structure best visualized on TEE. Short distance between the transducer and the posterior aspect of the heart enables higher-frequency transducers enhancing their ability to visualize cardiac structures with high spatial resolution and good temporal resolution. With TEE, the heart is not masked by extracardiac structures such as bones and lung tissue. Findings of an intracardiac spontaneous echo contrast or a thrombus on TTE should thus prompt a comprehensive TEE-guided assessment of the LA and the LAA. ${ }^{7}$ Best visualized in the midesophageal two-chamber view, the LAA should be assessed in a stepwise advancement of the omniplane angle with slight rotation of the probe. The LAA can also be visualized in the transgastric two-chamber view. TEE allows for optimal visualization of complete LA anatomy as well as functional assessment with Doppler interrogation. Role of echocardiography in such patients is not just to establish the existence of a thrombus/intracardiac mass source but also to determine nature, size, spatial orientation, mobility, fragility, and the stage of thrombus development (-Table 1). The aim is to ascertain likelihood of stroke or systemic embolism and also to guide appropriate therapy in any such patient. ${ }^{9}$

Initially, a thrombus is attached by a pedicle to the LAA and enlarges gradually in the LAA, acquiring the characteristic rounded appearance. Subsequent deposition of thrombotic material in layers over the initial thrombi contributes to the shape. Some LA thrombi (pedicled or early layered) are highly predisposed to dislodgement and are at extremely high risk of embolization. Besides physical properties of the clot, the LAA morphology also plays a key role in determining vulnerability to embolism. It has been categorized morphologically into four different subtypes: cactus, chicken wing, windsock, and cauliflower. The presence of chicken-wing LAA morphology makes patients less susceptible to thromboembolic events compared with those with other LAA morphologies. ${ }^{10}$ All this should be followed up with a close communication with the surgeon to minimize cardiac manipulation to avoid any dislodgment of the thrombus.

The surgeons aimed to minimize the cardiac manipulations to avoid the thrombus from dislodging. Following aortic cannulation, bicaval cannulation was planned in an attempt to minimize the manipulation of the heart. During the IVC cannulation, the authors observed the precise moment when the thrombus became detached from its anchorage on the atrial appendage wall, followed by its free floatation in the LA cavity. The mass did not prolapse between the mitral leaflets due to the tightly stenosed valve orifice. A giant, free-floating mass inside a cardiac cavity carries great risk of serious complications that include possible cerebral or peripheral embolic events; heart failure, due to impaired left ventricle filling; mitral regurgitation if the mass impedes the coaptation of the mitral valve leaflets; or syncope due to obstruction of the mitral valve orifice. ${ }^{11}$ Vigilance on the anesthesiologist's part and availability of real-time TEE lead to an instantaneous detection of the dislodged thrombus, followed by immediate corrective actions that avoided systemic embolization of a large thrombus.

The authors recommend that having completed the comprehensive TEE examination, all anesthesiologists must keep a strong vigil and review their echocardiographic findings, more so in patients with intracardiac masses, keeping an eye on them during any cardiac manipulation. Availability of realtime TEE during cardiac surgery has unscathed many lives from the perils of embolization. This case thus demonstrates that use of TEE is sine qua non for optimal patient outcome once the diagnosis of left atrial thrombi has been established.

\section{Conclusion}

This case demonstrates the steadfastness of intraoperative TEE in detecting LA/LAA masses and highlights its ability to alter surgical management toward a better patient outcome, thereby providing support for its routine use in patients with likelihood 
of LA/LAA masses. In the authors' opinion, TEE is an indispensable monitoring tool in patients with intracardiac thrombi, not just to confirm their presence but also to determine their vulnerability to dislodgement and embolization.

\section{References}

1 Tsioufis CP, Stefanadis CI, Tsiamis EG, Kallikazaros IE, Toutouzas PK. A free floating ball thrombus in the left atrial cavity. J Thorac Cardiovasc Surg 1999;118(06):1120-1122

2 Cakir O, Eren N, Oruç A, Buyukbayram H. Free-floating ball thrombus in the left atrium. Heart Vessels 2002;16(05):208-210

3 Misumi T, Kudo M, Ito T, Matsubara T, Kumamaru H. Floating ball thrombus in the left atrium with mitral stenosis. Jpn J Thorac Cardiovasc Surg 2003;51(08):387-389

4 Tornóczky T, Ajtay Z. Images in clinical medicine. Fatal freefloating left atrial thrombus. N Engl J Med 2004;351(27):e25

5 Parekh A, Jaladi R, Sharma S, Van Decker WA, Ezekowitz MD. Images in cardiovascular medicine. The case of a disappearing left atrial appendage thrombus: direct visualization of left atrial thrombus migration, captured by echocardiography, in a patient with atrial fibrillation, resulting in a stroke. Circulation 2006;114 (13):e513-e514

6 Leslie D, Hall TS, Goldstein S, Shindler D. Mural left atrial thrombus: a hidden danger accompanying cardiac surgery. J Cardiovasc Surg (Torino) 1998;39(05):649-650

7 Aschenberg W, Schlüter M, Kremer P, Schröder E, Siglow V, Bleifeld W. Transesophageal two-dimensional echocardiography for the detection of left atrial appendage thrombus. J Am Coll Cardiol 1986;7(01):163-166

8 Tahara A, Tahara N, Honda A, Imaizumi T. Mobile left atrial round mass free floating in mitral regurgitation flow mimicking a whale spray. BMJ Case Rep 2014;2014:bcr2013202725

9 Saric M, Armour AC, Arnaout MS, et al. Guidelines for the use of echocardiography in the evaluation of a cardiac source of embolism. J Am Soc Echocardiogr 2016;29(01):1-42

10 Di Biase L, Santangeli P, Anselmino M, et al. Does the left atrial appendage morphology correlate with the risk of stroke in patients with atrial fibrillation? Results from a multicenter study. J Am Coll Cardiol 2012;60(06):531-538

11 Guru R. Importance of transoesophageal echocardiography in preventing complications due to intraoperative dislodgement of left atrial thrombus. Indian J Anaesth 2010;54(06):577-579 